\title{
A Network Pharmacology Study to Uncover the Multiple Molecular Mechanism of the Chinese Patent Medicine Toujiequwen Granules in the Treatment of Corona Virus Disease 2019 (COVID-19)*
}

\author{
Bao-yu YANG ${ }^{1 \dagger}$, Hao-zhen $W_{A N G}{ }^{\dagger \dagger}$, Zhen-zhong MA ${ }^{1}$, Chen LU ${ }^{1}$, Yang LI ${ }^{1}$, Zi-yin LU1 ${ }^{1}$, Xiu-li LU ${ }^{1 \#}$, Bing GAO ${ }^{2 \#}$ \\ ${ }^{1}$ Department of Biochemistry and Molecular Biology, Life Science School, Liaoning University, Shenyang 110036, China \\ ${ }^{2}$ Department of Cell biology and Genetics, Shenyang Medical College, Shenyang 110034, China
}

(C) Huazhong University of Science and Technology 2021

\begin{abstract}
Summary: Since the outbreak of the novel corona virus disease 2019 (COVID-19) at the end of 2019, specific antiviral drugs have been lacking. A Chinese patent medicine Toujiequwen granules has been promoted in the treatment of COVID-19. The present study was designed to reveal the molecular mechanism of Toujiequwen granules against COVID-19. A network pharmacological method was applied to screen the main active ingredients of Toujiequwen granules. Network analysis of 149 active ingredients and 330 drug targets showed the most active ingredient interacting with many drug targets is quercetin. Drug targets most affected by the active ingredients were PTGS2, PTGS1, and DPP4. Drug target disease enrichment analysis showed drug targets were significantly enriched in cardiovascular diseases and digestive tract diseases. An "active ingredienttarget-disease" network showed that 57 active ingredients from Toujiequwen granules interacted with 15 key targets of COVID-19. There were 53 ingredients that could act on DPP4, suggesting that DPP4 may become a potential new key target for the treatment of COVID-19. GO analysis results showed that key targets were mainly enriched in the cellular response to lipopolysaccharide, cytokine activity and other functions. KEGG analysis showed they were mainly concentrated in viral protein interaction with cytokine and cytokine receptors and endocrine resistance pathway. The evidence suggests that Toujiequwen granules might play an effective role by improving the symptoms of underlying diseases in patients with COVID-19 and multi-target interventions against multiple signaling pathways related to the pathogenesis of COVID-19.
\end{abstract}

Key words: Toujiequwen granules; COVID-19; network pharmacology; target; molecular mechanism

Corona virus disease 2019 (COVID-19) caused by severe acute respiratory syndrome coronavirus 2 (SARS-CoV-2) (also known as 2019-nCoV) outbrokes in the world from December 2019. Till April 10, 2020, it had infected more than 80000 patients in China, resulting in more than 3000 deaths. More than 10000000 confirmed cases have been detected in more than 200 countries internationally. The World Health Organization (WHO) declared it as a global pandemic. The virus is contagious, thus threatening health and

Bao-yu YANG, E-mail: 928836176@qq.com; Hao-zhen WANG, E-mail: wanghzlnu@foxmail.com

${ }^{\dagger}$ The authors contributed equally to this study.

\#Corresponding authors, Xiu-li LU, E-mail: luxiuli@lnu.edu. cn; Bing GAO, E-mail: gaobingdr@hotmail.com

*The study was supported by the grants from the Education Department of Liaoning Province (No. LFW201701), Liaoning Provincial Key R \& D Project (No. 2020JH2/10300114) and Key Laboratory of Shenyang Science and Technology Bureau (No.18-007-0-02). causing harm to society. The International Committee on Taxonomy of Viruses (ICTV) announced the official English name of this new coronavirus as SARSCoV-2, while the WHO named the disease caused by the new coronavirus as COVID-19. SARS-CoV-2 and the viruses that caused severe acute respiratory syndrome (SARS) epidemic in 2003 and the Middle east respiratory syndrome (MERS) epidemic in 2012 belong to the coronavirus family, but they differ from each other. SARS-CoV-2 shares $79.5 \%$ of its genetic sequence with severe acute respiratory syndrome coronavirus (SARS-CoV) and has 96.2\% homology with bat coronavirus ${ }^{[1]}$. Phylogenetic analysis indicates that SARS-CoV-2 is close to a coronavirus in the horseshoe bat. At the same time, evolutionary analysis based on ORF1a/1b, S and N genes shows that SARS$\mathrm{CoV}-2$ is likely a new virus introduced from animals to humans independently ${ }^{[1]}$. Compared with SARS$\mathrm{COV}$ and MERS coronavirus (MERS-CoV), SARSCoV-2 has greater infectivity (higher R0) and lower 
mortality ${ }^{[2]}$. Although experts have proposed drug treatment programs to block the replication cycle and infection of COVID-19 and are currently performing clinical trials to test the efficacy of new antibodies, vaccines and drugs such as favipiravir, chloroquine phosphate and remdesivir, there is currently no specific medicine to treat COVID-19 ${ }^{[3]}$. Therefore, there is an urgent need for more effective and less toxic therapies against COVID-19.

Many clinical expert groups tried various Traditional Chinese Medicine (TCM) therapies or integrated Chinese and Western medicine treatments at the beginning of the pandemic and received good clinical outcome ${ }^{[4]}$. Patients with moderate and mild COVID-19 symptoms were easily cured with TCM treatment and the number of patients with moderate symptoms progressing to severe illness decreased significantly. For those with severe and critical illness, TCM is not just able to stabilize blood oxygen saturation, but also improves breathing difficulties. Toujiequwen granules (formerly known as Pneumonia Prescription No. 1 in Guangdong) was approved by the Guangdong Drug Administration for emergency approval, resulting in mass production. It is composed of 16 kinds of TCMs such as Forsythia suspensa, Cremastra appendiculata, Lonicera japonica, Scutellaria baicalensis, and Bupleurum chinense. According to the Guangdong Drug Administration website (http://mpa.gd.gov.cn/), 50 patients with COVID-19 pneumonitis (mild) were treated with Toujiequwen granules. At the 1st week, the temperature returned to normal in all the patients. Cough symptoms disappeared in $50 \%$ of the patients and sore throat symptoms in $52.4 \%$ of the patients. The symptoms of fatigue disappeared in $69.6 \%$ of the patients. The general symptoms were markedly alleviated, and no patients' conditions became severe. In clinical trials, COVID-19 patients were treated with Toujiequwen granules and Western medicine was also given. Early and timely use of Toujiequwen granules and Arbidol in 37 COVID-19 patients helped alleviate their symptoms. T cell counts were monitored and the immune function was restored ${ }^{[5]}$. In another clinical study, 10 days after COVID-19 patients were treated with Toujiequwen granules, the TCM syndrome score of the treatment group was significantly reduced $(P<0.05)$ and the absolute value of lymphocytes was significantly up-regulated. These studies suggest that the clinical symptoms and inflammation of COVID-19 can be alleviated by early and timely use of Toujiequwen granules $^{[6]}$. A few studies have shown that some of the Chinese medicines in the composition of Toujiequwen granules have antiviral effects. For example, forsythia and its main active ingredient, quercetin, have shown anti-human cytomegalovirus properties in in vitro studies $^{[7,8]}$. Honeysuckle is a common TCM which is used as antipyretic and antidote. Pharmacological studies have demonstrated that it can act against influenza A virus ${ }^{[9]}$. However, the detailed molecular mechanisms of the Toujiequwen granules in the treatment of COVID-19 is still unknown.

Network pharmacology is a study strategy that integrates high-throughput data analysis, molecular docking, drug-target network construction, and network feature analysis based on multi-target overall regulation ${ }^{[10,11]}$. It breaks the traditional concept of "one drug, one target, one disease" and provides new ideas and methods to research the "multi-ingredient, multitarget" approach of TCM and ethnic medicine ${ }^{[12,13]}$. Network pharmacology emphasizes multi-channel regulation of biological functions as well as signal pathways, improves the therapeutic effect of drugs, and reduces toxic and side effects, thereby improving the success rate of clinical trials of new drugs and laying a foundation for new drug development.

In this study, a network pharmacology method was used to systematically predict and screen potential active ingredients of Toujiequwen granules and key drug targets, and to analyze and study the mechanisms of the multiple-ingredients and multiple-targets of Toujiequwen granules against COVID-19. The underlying molecular mechanism of Toujiequwen granules against COVID-19 provides innovative medical and theoretical support to understand the positive effect of this prescription, and may facilitate its wide applications and development of new drugs based on Toujiequwen granules.

\section{MATERIALS AND METHODS}

\subsection{Screening of Active Ingredients and Corres- ponding Drug Targets of Toujiequwen Granules}

This study investigated the Chinese patent medicine Toujiequwen granules, which was produced by Guangdong E-Fang Pharmaceutical Co., Ltd. The TCMSP (Traditional Chinese Medicine Systems Pharmacology Database and Analysis Platform, http:// tcmspw.com/tcmsp.php) was used to retrieve the active ingredients, oral bioavailability $(\mathrm{OB} \geq 30 \%)$ and drug-like properties ( $\mathrm{DL} \geq 0.18$ ) as the conditions for screening the active ingredients of Toujiequwen granules. Among them, OB is an important indicator to evaluate a drug's efficacy ${ }^{[14]}$. DL is the similarity between a given ingredient and its known equivalent in Western medicine. Both factors are essential references to determine whether the TCM ingredients are active in the body. Toujiequwen granules are composed of 16 single TCMs, including Forsythia suspensa, Cremastra appendiculata, Lonicera japonica, Scutellaria baicalensis, Bupleurum chinense, Artemisia annua, Angelica decursiva, Fritillaria cirrhosa, Prunus mume, Scrophularia ningpoensis, Atractylodes lancea, Astragalus mongholicus, 
Pseudostellaria heterophylla, Poria cocos, Cicadae periostracum and Ground beetles. The active ingredient of every TCM ingredient in Toujiequwen granules was retrieved. A total of 149 ingredients were obtained after removing redundancies. A total of 330 drug targets (proteins) corresponding to those 149 ingredients were identified by searching the TCMSP database. The bioDBnet online tool (https://biodbnetabcc.ncifcrf.gov/db/db2db.php) was used to change the protein names of drug target to human gene names. Some of the drug targets corresponding to each active ingredient in TCMSP database were obtained from DrugBank (https://www.drugbank.ca/), and some of the drug targets verified by experiments came from the HIT database (http://bigd.big.ac.cn/databasecommons/ database/id/690). Unverified drug targets for the active ingredients were predicted by the SysDT model. The database of active ingredients and their corresponding drug targets (ingredients-drug target) was constructed. Cytoscape 3.6.1 software $^{[15]}$ was used to construct an active ingredient-target network to analyze the synergistic relationship between the active ingredients and multiple effective drug targets.

\subsection{Enrichment Disease Analysis of Drug Target}

The gene names of 330 drug targets matching the active ingredients of Toujiequwen granules were imported into the CTD online analysis platform (http://ctdbase.org) database. The results of disease enrichment analysis corresponding to these drug targets were obtained. This enabled the analysis of the possible role of Toujiequwen granules in treating the underlying disease in patients with COVID-19.

\subsection{Prediction of Potential Disease Targets of COVID-19}

The DisGeNET (http://www.disgenet.org/) disease target prediction analysis platform was utilized to predict disease targets of COVID-19. The potential targets in respiratory diseases and viral diseases were screened. Because of similarities in the RNA sequences of SARS-CoV and SARS-CoV-2 and similarities in the relative symptoms, especially in severe cases, SARS-CoV was used to predict the disease targets of COVID-19 ${ }^{[16]}$. In total, 84 drug targets of Toujiequwen granules were selected and a COVID-19 disease targets database was created.

1.4 Construction of a Network of the Active Ingredients of Toujiequwen Granules, Disease Targets and COVID-19

The database of ingredients and drug targets, including 149 active ingredients of Toujiequwen granules and 330 predicted drug target genes, in addition to the database of COVID-19 disease targets were merged by Cytoscape3.6.1 software to construct an active ingredient-key targets-COVID-19 network. Overlap targets of drug targets of active ingredients and disease targets of COVID-19 were called as key targets for Toujiequwen granules against COVID-19.

\subsection{GO Function Enrichment Analysis and KEGG} Pathway Enrichment Analysis

$\mathrm{R}$ language package clusterProfiler ${ }^{[17]}$ was utilized to perform GO and KEGG analysis on key targets. GO enrichment analysis includes gene ontology biological process (GO-BP) analysis, gene ontology cellular ingredient (GO-CC) analysis, gene ontologymolecular function (GO-MF) analysis of biological molecules. Significant enrichment conditions for GO and KEGG were set as $P$-value $<0.01$ and FDR $<0.2$. Enrichment results were sorted according to their statistical significance ( $P$ value). The top 20 enrichment results for each enrichment analysis were selected for histogram and bubble chart.

\section{RESULTS}

\subsection{Screening of Candidate Ingredients and Acquisition of Targets}

First, 16 TCMs present in Toujiequwen granules were submitted to the TCMSP website to search for active ingredients under the conditions of $\mathrm{OB} \geq 30 \%$ and $\mathrm{DL} \geq 0.18$. We obtained 9 in Atractylodes lancea, 17 in Bupleurum chinense, 13 in Fritillaria cirrhosa, 15 in Poria cocos, 36 in Scutellaria baicalensis, 20 in Astragalus mongholicus, 23 in Lonicera japonica, 23 in Forsythia suspensa, 24 in Angelica decursiva, 22 in Artemisia annua, 3 in Cremastra appendiculata, 8 in Pseudostellaria heterophylla, 8 in Prunus mume, and 9 in Pcrophularia ningpoensis. Cicadae periostracum and ground beetle had no active ingredients that met the screening conditions. A total of 149 compounds were collected after removing the redundant active ingredients. Then 149 active ingredient compounds were matched in the TCMSP database to find relevant drug targets. A total of 330 drug targets were obtained after removing redundancy.

\subsection{Feature Analysis of Active Ingredient-drug Target Network}

The 149 active ingredients and their corresponding 330 drug targets were constructed into a network map by Cytoscape software version 3.6.1. We plotted the top $10 \%$ of the most active ingredients and drug targets according to their high degree and betweenness centrality. The result is depicted in fig. 1, including its 62 nodes and 1056 edges. The degree and betweenness are positively related to the importance of the active ingredient and drug target. The red circle represents the active ingredient, the blue triangle represents the drug target, and the size of the node represents the magnitude of its degree. We observed that many ingredients interacted with multiple drug targets, and many drug targets were hit by multiple active ingredients. Among them, the most important compound was determined to be MOL000098 (quercetin) with 189 
edges, representing that 189 drug targets were hit by this active ingredient. It pointed out that quercetin has a wide range of physiological activities, such as anti-oxidative, anti-inflammatory, and anti-tumor properties $^{[7]}$. Active ingredients that interact most with drug targets also included luteolin, kaempferol, wogonin, formononetin, sagerin, beta-sitosterol, tanshinone IIA, and baicalein. Top 10 drug targets modulated by multiple active ingredients were PTGS2, PTGS1, NCOA2, DPP4, SCN5A, AR, PRSS1, F2, NOS and PRKCA. PTGS2 and PTGS1 are prostaglandin endoperoxide synthases as well as rate-limiting enzymes involved in the synthesis of prostaglandins. Prostaglandins participate in the pathological processes of various inflammatory reactions, cancers, and assorted cardiovascular diseases ${ }^{[18]}$. Dipeptidyl peptidase 4 or DPP4, also known as T cell surface antigen CD26, is a serine protease on the cell surface. The most widely studied protein substrate of DPP4 is GLP-1. GLP-1 can lower blood sugar by stimulating insulin and inhibiting glycogen. Therefore, DPP4 inhibitors have become one of the popular drugs for the treatment of diabetes ${ }^{[19]}$. Additionally, a recent study revealed that DPP4 secreted by the liver may enter the bloodstream and then activate inflammatory cells (macrophages) ${ }^{[20]}$. These data implied that Toujiequwen granules might have multi-target synergistic effects on the human body.

\subsection{Analysis of Enriching Diseases of Drug Targets of the Active Ingredients from Toujiequwen Granules}

Some reports have shown that elderly males with underlying conditions or chronic diseases are at risk for severe disease. Pre-existing medical conditions such as diabetes, hypertension, heart disease and kidney disease can increase susceptibility to COVID-19. For instance, a study by Wang's team at Suzhou Hospital Affiliated to Nanjing Medical University recently found that SARS-CoV-2 may infect and damage male testes. Recently, scientists from the National Institutes of Health $(\mathrm{NIH})$ have come to a similar

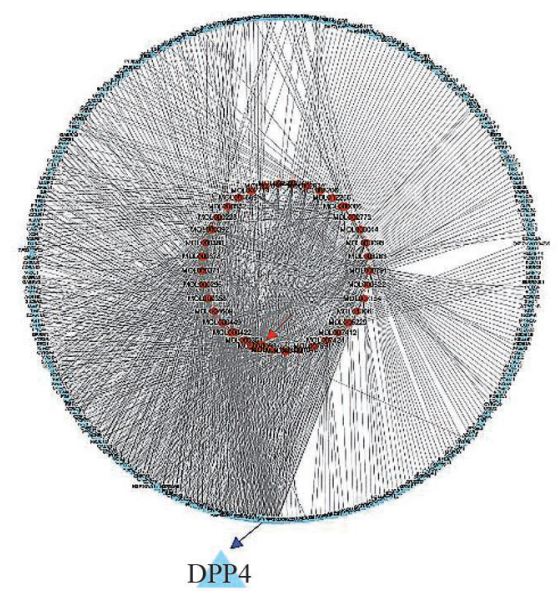

conclusion $^{[21]}$. Further, Shanhong's research group at Sun Yat-sen University found that the SARS-CoV-2 receptor ACE-2 was expressed in patient's stomach, duodenum, and rectal glandular epithelial cells ${ }^{[22]}$. SARS-CoV-2 nucleocapsid protein was detected in glandular epithelial cells of these tissues. The digestive tract can be infected with SARS-CoV-2 ${ }^{[22]}$. One study suggested that SARS-CoV-2 could also infect the nervous system ${ }^{[23]}$. Altogether these studies suggest that the attacks of SARS-CoV-2 on these tissues may exacerbate patients' underlying diseases.

In order to understand which underlying diseases could be affected by Toujiequwen granules in the human body, we performed an enrichment analysis involving diseases of the 330 drug targets of Toujiequwen granules on the CTD online analysis platform. Top ten diseases following enrichment analysis are shown in table 1. We found that the drug targets of Toujiequwen granules can be highly enriched in cardiovascular diseases, neurological diseases, digestive diseases, respiratory diseases, male urogenital diseases, and other conditions. These enriched diseases affected by Toujiequwen granules overlap with the underlying diseases of COVID-19 patients. Our results suggested that Toujiequwen granules can improve symptoms of the underlying diseases in patients with COVID-19, and may therefore play a role in delaying or reducing disease progression.

\subsection{Prediction of Disease Target Database of COVID-19}

In order to figure out how Toujiequwen granules directly affect the drug target of COVID-19, we constructed a drug target database of COVID-19 in DisGeNET (https://www.disgenet.org/). The first study of postmortem biopsies of a COVID-19 patient revealed the pathological features of COVID-19 greatly resemble those seen in SARS and MERS coronavirus infection $^{[16]}$. The SARS-CoV-2 and SARS viruses have similarities with respect to the whole genome sequence $(79 \%)^{[24]}$ and they share the same receptor, ACE2.

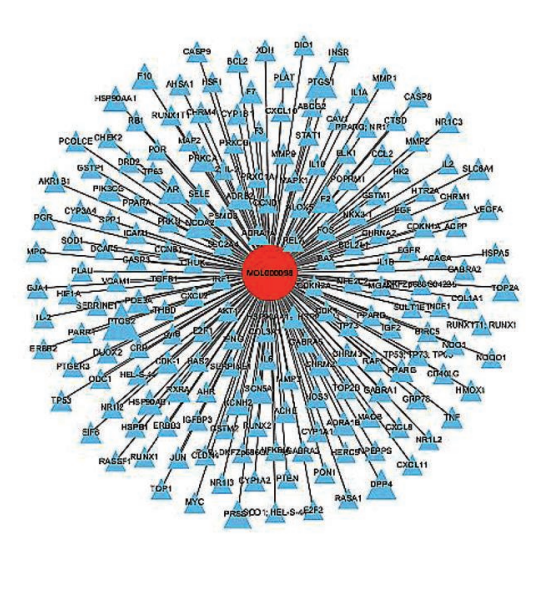

Fig. 1 Network model of active ingredients-drug target

Red plots represent active ingredients contained in Toujiequwen granules. Blue plots represent active ingredients targets. 
Table 1 Top 10 diseases affected by drug targets of the active ingredients from Toujiequwen granules in CTD enrichment analysis

\begin{tabular}{lccc}
\hline Disease name & Disease ID & Corrected $P$ value & Annotated genes quantity \\
\hline Pathological conditions, signs and symptoms & MESH:D013568 & $7.38 \mathrm{E}-169$ & 216 \\
Cardiovascular diseases & MESH:D002318 & $9.84 \mathrm{E}-160$ & 165 \\
Vascular diseases & MESH:D014652 & $8.07 \mathrm{E}-154$ & 142 \\
Pathologic processes & MESH:D010335 & $8.78 \mathrm{E}-148$ & 180 \\
Neoplasms by site & MESH:D009371 & $2.66 \mathrm{E}-143$ & 190 \\
Neoplasms & MESH:D009369 & $5.55 \mathrm{E}-143$ & 204 \\
Digestive system diseases & MESH:D004066 & $3.16 \mathrm{E}-121$ & 171 \\
Nervous system diseases & MESH:D009422 & $8.53 \mathrm{E}-121$ & 168 \\
Respiratory tract diseases & MESH:D012140 & $8.45 \mathrm{E}-117$ & 123 \\
Male urogenital diseases & MESH:D052801 & $9.39 \mathrm{E}-115$ & 137 \\
\hline
\end{tabular}

Latest research shows that they also have similarities in the crystal structure of the resolved protein (such as in Mpro protease and S protein) ${ }^{[2]}$. Although four of the five key amino acids in the $\mathrm{S}$ protein that binds to ACE2 protein is different in SARS-CoV-2, these different amino acids have perfectly maintained their interaction with $\mathrm{ACE} 2^{[25]}$. Based on the above reasons, we selected the disease targets of SARS to construct the target library of COVID-19 in the DisGeNET platform. A total of 84 disease targets were predicted as disease targets in the COVID-19 database.

\subsection{Analysis of Characteristics in Active Ingredient-} disease Target-COVID-19 Network

The active ingredient-drug target database and the COVID-19 disease target library were merged using Cytoscape software 3.6.1 to generate the active ingredient-disease target-COVID-19 network (fig. 2) and related analysis was performed (tables 2 and 3 ).

A total of 57 of 149 active ingredients contained in Toujiequwen granules may affect COVID-19 by interacting with 15 disease targets of COVID-19 including quercetin, luteolin, wogonin, kaempferol, and other compounds. The 15 disease targets include DPP4, MAPK14, TNF, and IL-6. Interestingly, DPP4 can interact with up to 54 of the 57 related active ingredients, suggesting that DPP4 may be a new potential key target for Toujiequwen granules in the treatment of COVID-19. This result indicated that further research could be focused on the infected ratio in the population of COVID-19 patients with diabetes and who are taking DPP4 inhibitors, as well as the proportion of mild and severe cases. These investigations may suggest whether it is clinically possible to use potent DPP4 inhibitors such as sitagliptin and saxagliptin as

Table 2 The possible main active ingredient of Toujiequwen granules against COVID-19

\begin{tabular}{|c|c|c|c|c|}
\hline MOLNAME & Ingredient & Medicine & Degree & Target \\
\hline MOL000098 & Quercetin & $\begin{array}{c}\text { Lianqiao, Jinyinhua, Huangqi, } \\
\text { Wumei, Qianhu, Qinghao, Chaihu }\end{array}$ & 13 & $\begin{array}{l}\text { DPP4, CCL2, CXCL8, TNF, BCL2L1, } \\
\text { MAPK1, CXCL10, IL2, IL6, IL10, } \\
\text { RB1, IFNG, AHSA1 }\end{array}$ \\
\hline MOL000006 & Luteolin & $\begin{array}{c}\text { Lianqiao, Jinyinhua, Taizishen, } \\
\text { Qinghao }\end{array}$ & 10 & $\begin{array}{l}\text { DPP4, CCL2, IL6, BCL2L1, MAPK1, } \\
\text { IL10, RB1, IFNG, TNF, IL2, }\end{array}$ \\
\hline MOL000173 & Wogonin & Lianqiao, Huangqin, Cangzhu & 8 & $\begin{array}{l}\text { CCL2, MAPK14, FN1, TNF, IL6, } \\
\text { CXCL8, DPP4, AHSA1 }\end{array}$ \\
\hline MOL000422 & kaempferol & $\begin{array}{l}\text { Lianqiao, Jinyinhua, Huangqi, } \\
\text { Wumei, Qinghao, Chaihu }\end{array}$ & 3 & DPP4, TNF, AHSA1 \\
\hline MOL000378 & 7-O-methylisomucronulatol & Huangqi & 2 & DPP4, MAPK14 \\
\hline MOL000392 & Formononetin & Huangqi & 2 & DPP4, MAPK14 \\
\hline MOL000354 & Isorhamnetin & Huangqi, Qinghao, Chaihu & 2 & DPP4, MAPK14 \\
\hline MOL007154 & Tanshinone iia & Qianhu & 2 & DPP4, AHSA1 \\
\hline MOL007991 & $\begin{array}{l}\text { 2-Methoxy-9,10- } \\
\text { dihydrophenanthrene-4,5-diol }\end{array}$ & Shancigu & 2 & DPP4, MAPK14 \\
\hline MOL003095 & $\begin{array}{l}\text { 5-Hydroxy-7-methoxy-2-( } 3,4,5- \\
\text { trimethoxyphenyl)chromone }\end{array}$ & Jinyinhua & 2 & DPP4, MAPK14 \\
\hline MOL005229 & Artemetin & Qinghao & 2 & DPP4, MAPK14 \\
\hline MOL002928 & Oroxylin a & Huangqin & 2 & DPP4, IL6 \\
\hline MOL008206 & Moslosooflavone & Huangqin & 2 & DPP4, MAPK14 \\
\hline MOL003044 & Chryseriol & Jinyinhua & 2 & DPP4, MAPK14 \\
\hline MOL002933 & 5,7,4'-Trihydroxy-8-methoxyflavone & Huangqin & 2 & DPP4, MAPK14 \\
\hline MOL004083 & Tamarixetin & Qinghao & 2 & DPP4, MAPK14 \\
\hline MOL007423 & 6,8-Di-c-glucosylapigenin_qt & Qinghao & 2 & DPP4, MAPK14 \\
\hline MOL007404 & Vitexin_qt & Qinghao & 2 & DPP4, MAPK14 \\
\hline
\end{tabular}


Table 3 The main possible disease targets of COVID-19 affected by Toujiequwen granules

\begin{tabular}{llc}
\hline Number & Gene name & Degree \\
\hline 1 & DPP4 & 53 \\
2 & MAPK14 & 16 \\
3 & TNF & 7 \\
4 & IL6 & 5 \\
5 & AHSA1 & 5 \\
6 & CCL2 & 4 \\
7 & IFNG & 3 \\
8 & IL2 & 3 \\
9 & CXCL8 & 3 \\
10 & BCL2L1 & 3 \\
11 & MAPK1 & 3 \\
12 & IL10 & 3 \\
13 & RB1 & 3 \\
14 & CXCL10 & 2 \\
15 & FN1 & 2 \\
\hline
\end{tabular}

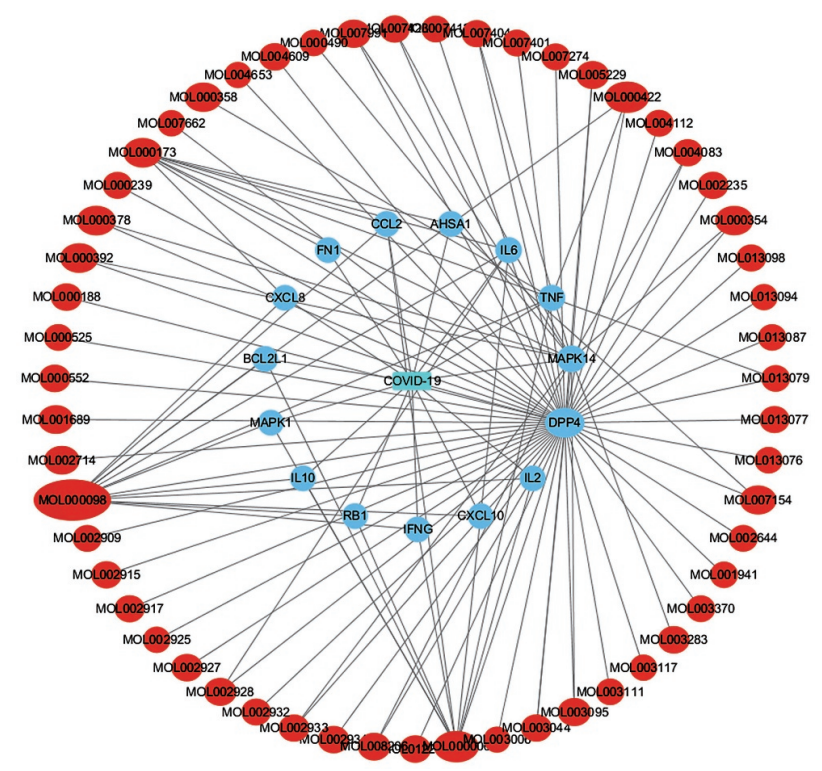

Fig. 2 Active ingredients-disease target-disease network The size of the graphic area indicates the importance of the nodes in the network. Red plots represent active ingredients contained in Toujiequwen granules related to COVID-19. Blue plots represent active ingredients targets which relate to Toujiequwen granules and COVID-19.

one of the treatment strategies for COVID-19.

\subsection{GO Function Enrichment Analysis}

In order to determine the gene enrichment of the 15 key disease targets of COVID-19 affected by Toujiequwen granules, GO analysis was performed. $\mathrm{GO}$ analysis including functional enrichment of target genes is based on two parameters: $P$ value $(P<0.01)$ and false discovery rate (FDR) $<0.2$. Results obtained from the functions of enrichment or the top 20 (when the qualified enrichment results are more than 20) were analyzed, as shown in figure 3 . The study found that the top 20 biological processes (BP) enriched by these 15 potential disease targets mainly included lipopolysaccharide (LPS), cell response process, bacterial molecular response process, biological stimulus response process, multiple cell-cell adhesion regulation and protein secretion, peptide secretion regulation and other processes. Enriched cellular components (CC) are related to several cell membranerelated components, secreted particles, vesicles, and so on. Enriched protein molecular functions (MF) mainly include cytokine activity, cytokine receptor binding, receptor ligand activity, chemokine activity, G-protein coupled receptor binding, and MAP kinase activity. The enrichment results on gene functions suggested that Toujiequwen granules may achieve their therapeutic effect on COVID-19 by mainly acting on inflammation-related cellular response processes and protein targets with cytokine-related functions.

\subsection{KEGG Pathway Enrichment Analysis}

In order to determine the main signaling pathways involved in the treatment of COVID-19 with Toujiequwen granules, signaling pathways enriched with 15 key disease targets were performed. For the study, top 20 significantly enriched KEGG signaling pathways were identified and selected for analysis (fig. 4). It can be seen from the figure that the enriched cell signaling pathways mainly include viral proteincytokine and cytokine receptor interaction pathways, cytokine-cytokine receptor interaction pathways, cell aging signaling pathways, chemokine signaling pathways, TGF- $\beta$ signaling pathway, PI3K-Akt signaling pathway, and endocrine resistance signaling pathway. The evidence suggests that the active ingredients of Toujiequwen granules can alleviate lung inflammation and improve the symptoms of COVID-19 mainly through cytokines, inflammationrelated signaling pathways, and viral infection-related signaling pathways.

\section{DISCUSSION}

Since the outbreak of COVID-19 at the end of 2019 , the virus has been proved to be contagious and threatened human health. The pandemic has not yet ended, and medical professionals, pharmacologists, and biologists all over the world have devoted their efforts to fight against this virus. The latest research on SARSCov-2 shows that the disease development is related to blood biochemical indicators, such as cytokines and C-reactive protein (CRP) ${ }^{[26]}$, which can potentially be used as diagnostic markers for COVID-19. A few cytokines are directly related to viral load and lung injury, and the factors affecting these signal mediators can therefore be potential drugs targets ${ }^{[26]}$. Specific antiviral drugs (small molecule monomer compounds), antibodies and vaccines against SARS-Cov-2 have not been officially approved yet due to the specific pathogenicity and transmission mechanism of the virus. 
A BP: biological process
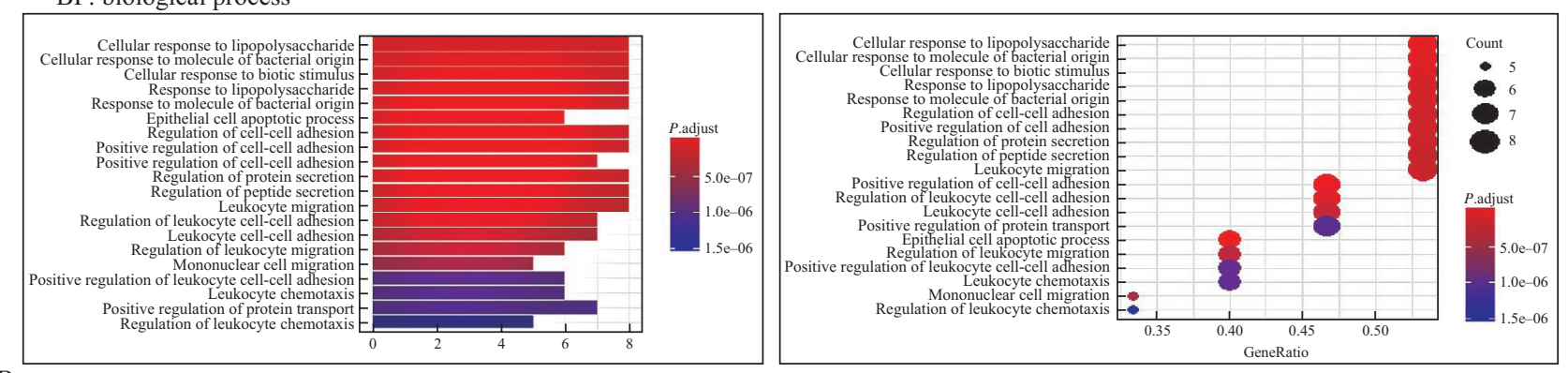

B CC: cellular components
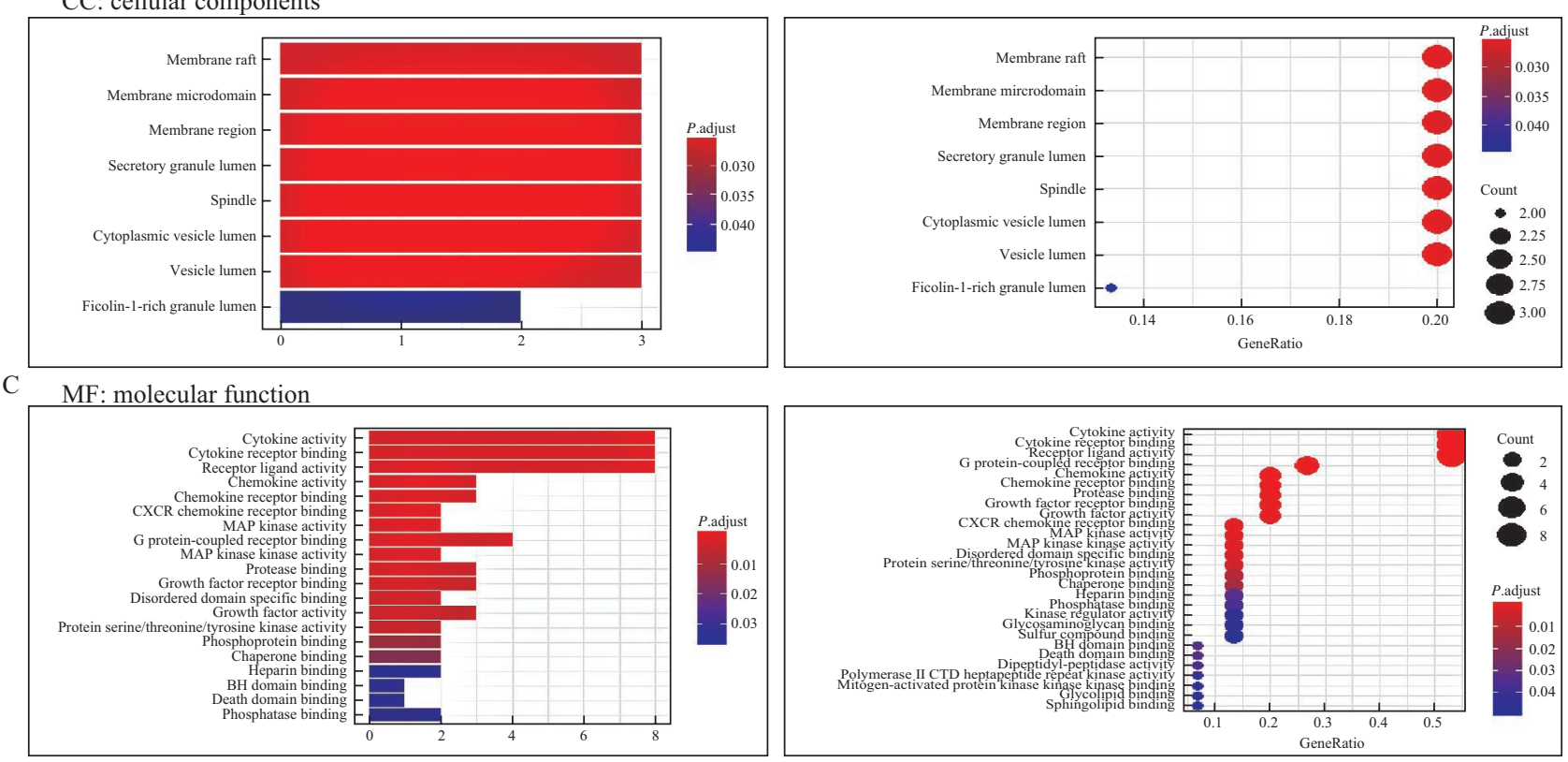

Fig. 3 Analysis of GO enrichment results of Toujiequwen granules related to the possible disease targets of COVID-19

The $y$-axis shows significantly enriched GO categories of the target genes, and the $x$-axis shows counts of targets or GeneRatio $(\mathrm{FDR}<0.02)$.
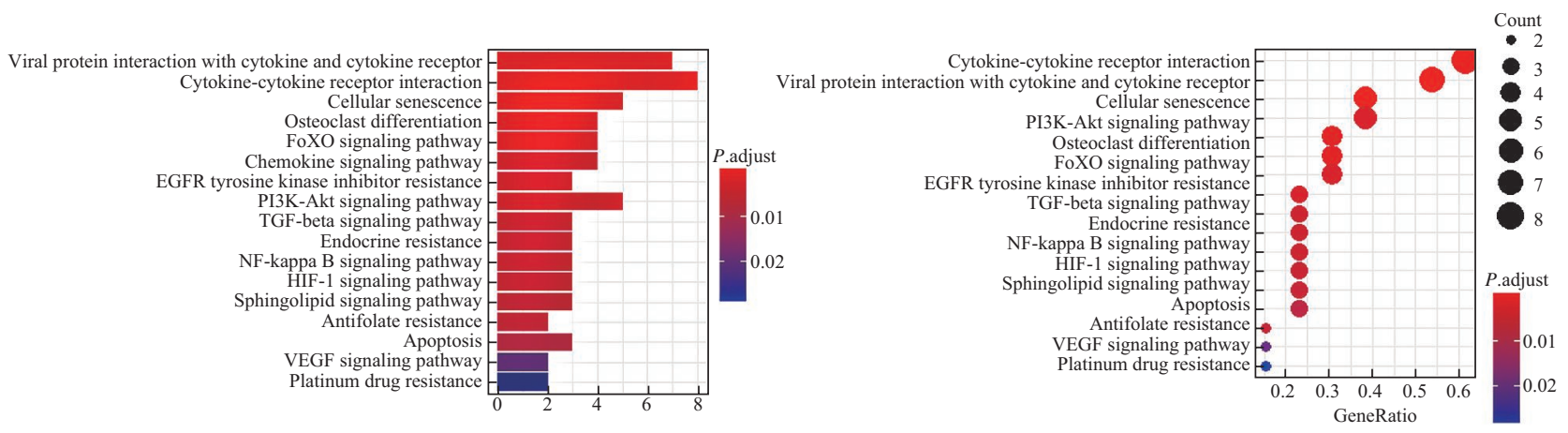

Fig. 4 KEGG enrichment analysis for COVID-19 targets of Toujiequwen granules

The Y-axis shows significantly enriched KEGG pathways of target genes, and the $x$-axis shows the GeneRatio (FDR $<0.02)$. GeneRatio stands for the ratio of the number of target genes belonging to a pathway to the number of all the annotated genes located in the pathway. Higher GeneRatio represents higher level of enrichment. The size of the dot indicates the number of target genes in the pathway, and the color of the dot reflects the different values of $P$ values.

On the other hand, scientists are trying to use existing drugs and performing preclinical and clinical trials of drugs such as favipiravir, chloroquine phosphate, and remdesivir. However, there are no approved drugs specifically against SARS-Cov-2 and COVID-19 so far. At the same time, many medical teams in China have received better clinical effects by utilizing TCM or combination of Chinese traditional and Western medicine.

In this study, a network pharmacology method was applied to analyze the main active ingredients as well as effective targets of the Chinese patent medicine Toujiequwen granules for the treatment of COVID-19. The network analysis was combined with related 
disease targets of SARS. GO and KEGG enrichment analysis with the key drug target of ingredienttarget-disease network has been further resolved. This study systematically explored the molecular mechanism of Toujiequwen granules for the treatment of COVID-19. We found that pharmacological ingredients in Toujiequwen granules, such as quercetin, luteolin, kaempferol, and baicalein, may be involved in inflammation, cancer, and various cardiovascular diseases based on the analysis of the active ingredienttarget disease network. The pathological process targeted by the active ingredients may effectively improve the symptoms of underlying diseases in patients with COVID-19, thereby delaying or reducing the disease process. We also found that DPP4, a key target in the network, can interact with up to 54 of 57 related active ingredients, suggesting that DPP4 may potentially be the most important target for the treatment of COVID-19 with Toujiequwen granules (fig. 1). Furthermore, we suggest that DPP4 could become a new key target for the prevention and treatment of COVID-19 by utilizing the existing inhibitors. In addition, Toujiequwen granules may play a role in improving symptoms of patients with COVID-19 by affecting the cell cycle, regulating immunity, inflammation, and adjusting cell metabolism (fig. 3). Studies showed that patients severely affected by COVID-19 were more prone to neurological symptoms, especially acute cerebrovascular disease, disturbance of consciousness, and muscle damage ${ }^{[23]}$. Some studies found that men were more susceptible to COVID-19 than women, and their symptoms are more severe after infection $^{[16]}$. This is consistent with our enrichment analysis of drug targets (table 1). Taken together, these results suggested that Toujiequwen granules might play an effective role by improving the underlying disease symptoms of patients with COVID-19. It may also act by multiple-targets intervention and act against multiple signaling pathways that are responsible for the disease caused by SARS-CoV-2. Our results provide important theoretical support for TCM therapy against COVID-19, in addition to providing new ideas and references for treatments by combination of TCM and Western medicine against COVID-19.

In the present study, we only used bioinformatics database mining and bioinformatics software analysis to predict the possible molecular mechanism of Toujiequwen granule against COVID-19. Further research is required at the cellular and animal levels to study detailed molecular mechanisms. This study attempts to predict and explore the molecular mechanism of Toujiequwen granules against COVID-19 from the perspective of modern medicine, which may help to understand how Toujiequwen granules and other traditional Chinese prescriptions work to cure COVID-19. We hope our data may facilitate wider use of TCM in the treatment of COVID-19 especially since there are no specific anti-viral drugs or vaccines available.

\section{Conflict of Interest Statement} interest.

The authors declare that they have no conflicts of

\section{REFERENCES}

1 Rio CD, Malani PN. 2019 Novel Coronavirus-Important Information for Clinicians. JAMA, 2020,323(11):10391040

2 Wrapp D, Wang NS, Corbett KS, et al. Cryo-EM structure of the 2019-nCoV spike in the prefusion conformation. Science, 2020,367(6483):1260-1263

3 Zumla A, Chan J, Azhar E, et al. Coronaviruses - drug discovery and therapeutic options. Nat Rev Drug Discov, 2016,15(5):327-347

4 Gao Y, Qiu HB, Zhou S, et al. Accumulated Clinical Experiences from Successful Treatment of 1377 Severe and Critically Ill COVID-19 Cases. Curr Med Sci, 2020, 40(4):597-601

5 Fu X, Lin L, Tan X. Clinical study on 37 case of COVID-19 treated with integrated traditional Chinese and Western Medicine. Zhongyao Xinyao yu Linchuang Yaoli (Chinese), 2020,31(5):600-604

6 Fu X, Lin L, Tan X. Clinical Study on Treatment of Cases of COVID-19 with Toujie Quwen Granules. Zhongguo Shiyan Fangjixue Zazhi (Chinese), 2020,26(12):44-48

7 Sohn EJ, Kim JM, Kang SH, et al. Restoring Effects of Natural Anti-Oxidant Quercetin on Cellular Senescent Human Dermal Fibroblasts. Am J Chinese Med, 2018, 46(4):853-873

8 Zhang D, Fang J, Chen J, et al. An in vitro study of antihuman cytomegalovirus effect of Forsythia suspensa and its main active ingredient quercetin. Zhongguo Zhongyao Zazhi (Chinese), 2010,35(8):1055-1059

9 Zhang ZM, Guo SJ, Fu SJ, et al. Research Progress of Active Ingredients and Pharmacological Action of Honeysuckle. Animal Husbandry Feed Sci, 2014(1):2224

10 Sun Y, Xiong YY, Wu HZ, et al. Active Ingredients and Mechanism of Action of Rhizoma Coptidis against Type 2 Diabetes Based on Network-Pharmacology and Bioinformatics. Curr Med Sci, 2020,40(2):257-264

11 Xiong $\mathrm{WC}, \mathrm{Wu} \mathrm{HZ}$, Xiong YY, et al. Network Pharmacology-based Research of Active Components of Albiziae Flos and Mechanisms of Its Antidepressant Effect. Curr Med Sci, 2020,40(1):123-129

12 Hopkins A. Network pharmacology: the next paradigm in drug discovery. Nat Chem Biol, 2008,4(11):682-690

13 Liu XK, Wu JR, Zhang D, et al. A Network Pharmacology Approach to Uncover the Multiple Mechanisms of Willd. on Colorectal Cancer. Evid Based Complement Alternat Med, 2018, 2018:1-12

14 Liu J, Pei M, Zheng C, et al. A Systems-Pharmacology Analysis of Herbal Medicines Used in Health Improvement Treatment: Predicting Potential New Drugs and Targets. Evid Based Complement Alternat Med, 2013,2013(6):938764

15 Shannon P, Markiel A, Ozier O, et al. Cytoscape: 
a software environment for integrated models of biomolecular interaction networks. Genome Res, 2003, 13(11):2498-2504

$16 \mathrm{Xu} \mathrm{Z}$, Shi L, Wang YJ, et al. Pathological findings of COVID-19 associated with acute respiratory distress syndrome. Lancet Respir Med, 2020,8(4):420-422

17 Yu GC, Wang LG, Han YY, et al. clusterProfiler: an $\mathrm{R}$ Package for Comparing Biological Themes Among Gene Clusters. Omics, 2012,16(5):284-287

$18 \mathrm{Xu} \mathrm{H}$. Research progress of prostaglandin receptors and related drugs. J China Pharm Univ, 2010,41(5):385-394

19 Mcdougall C, Mckay GA, Fisher M. Drugs for diabetes: Part 5 DPP-4 inhibitors. Br J Cardiol, 2011,18(3):130132

20 Kitano, H. Systems Biology: A Brief Overview. Science, 2002,295(5560):1662-1664

21 Wang ZP, Xu XJ. scRNA-seq Profiling of Human Testes Reveals the Presence of ACE2 Receptor, a Target for SARS-CoV-2 Infection, in Spermatogonia, Leydig and Sertoli Cells. Cell, 2020,9(4):920
22 Xiao F, Tang MW, Zheng XB, et al. Evidence for gastrointestinal infection of SARS-CoV-2. Gastroenterology, 2020,158(6):1831-1833

23 Mao L, Jin HJ, Wang MD, et al. Neurologic Manifestations of Hospitalized Patients with Coronavirus Disease 2019 in Wuhan, China. JAMA Neurol, 2020,77(6):683-690

24 Zhi P, Yang XL, Wang XG, et al. A pneumonia outbreak associated with a new coronavirus of probable bat origin. Nature, 2020,579(7798):270-273

$25 \mathrm{Xu} \mathrm{XT}$, Chen P, Wang JF, et al. Evolution of the novel coronavirus from the ongoing Wuhan outbreak and modeling of its spike protein for risk of human transmission. Sci China Life Sci, 2020,63(3):457-460

26 Liu YX, Yang Y, Zhang C, et al. Clinical and biochemical indexes from 2019-nCoV infected patients linked to viral loads and lung injury. Sci China Life Sci, 2020,63(3):364-374

(Received Jul. 8, 2020; accepted Nov. 13, 2020) 